\title{
Use of DNA flow cytometry in the diagnosis, risk stratification, and management of gastric epithelial dysplasia
}

\author{
Kwun Wah Wen ${ }^{1} \cdot$ Peter S. Rabinovitch ${ }^{2} \cdot$ Danning Huang $^{3} \cdot$ Aras N. Mattis $^{1} \cdot$ Gregory Y. Lauwers $^{4} \cdot$ Won-Tak Choi $^{1}$
}

Received: 1 February 2018 / Revised: 3 March 2018 / Accepted: 4 March 2018 / Published online: 22 May 2018

(c) United States \& Canadian Academy of Pathology 2018

\begin{abstract}
The natural history of gastric epithelial dysplasia and the consequential surveillance strategies are not well defined in the West. To date, the diagnosis relies on morphology, and no reliable adjunct methods, either immunohistochemical or molecular, have reproducibly been able to confirm the diagnosis and/or risk stratify gastric epithelial dysplasia. Yet, such a tool would be useful in confirming the diagnosis, and developing objective and rational surveillance guidelines. DNA flow cytometry was performed using formalin-fixed paraffin-embedded gastric tissue from 23 cases of high-grade dysplasia and 38 cases of lowgrade dysplasia. Twenty-four benign background mucosal samples from the same cohort (20 biopsies and 4 surgical resections from 16 low- and 8 high-grade dysplasia cases) were utilized as controls. The presence of DNA content abnormality (aneuploidy or elevated $4 \mathrm{~N}$ fraction) correlated with increasing levels of dysplasia, as DNA content abnormality was detected in 18 (78\%) of 23 high-grade dysplasia, 5 (13\%) of 38 low-grade dysplasia, and none of 24 non-dysplastic samples. 1 and 4-year detection rates of high-grade dysplasia or gastric adenocarcinoma in low-grade dysplasia patients with DNA content abnormality were $80 \%(p=0.003)$ and $100 \%(p=0.005)$, respectively, whereas patients with low-grade dysplasia but with normal DNA content had 1, 4, and 12-year detection rates of 23, 32, and 54\%, respectively. The univariate hazard ratio (HR) for subsequent detection of high-grade dysplasia or gastric adenocarcinoma in low-grade dysplasia patients with DNA content abnormality was $6.9(p=0.001)$. Older patients $(\mathrm{HR}=1.1, p=0.005)$ and those with familial adenomatous polyposis $(\mathrm{HR}=9.7, p=0.029)$ also had an increased risk for developing high-grade dysplasia or gastric adenocarcinoma in the univariate analysis, but only DNA content abnormality demonstrated a significantly elevated HR of 5.9 in the multivariate analysis $(p=0.005)$. While older age showed a minimally elevated risk ( $\mathrm{HR}=1.1, p=0.013)$, no other potential risk factors, including male gender, ethnicity, polypoid endoscopic appearance, Helicobacter pylori infection, and intestinal metaplasia, were significantly associated with subsequent detection of high-grade dysplasia or gastric adenocarcinoma in the multivariate analysis. Among the 18 high-grade dysplasia cases with DNA content abnormality, 13 cases (72\%) developed gastric adenocarcinoma within a mean follow-up time of 9 months, conferring a HR of 2.5; however, this did not reach statistical significance. In conclusion, the presence of DNA content abnormality can identify a subset of lowgrade dysplasia patients who are at increased risk for subsequent detection of high-grade dysplasia or gastric adenocarcinoma. It can also provide confirmatory evidence to a morphologic impression or suspicion of high-grade dysplasia. The majority of gastric epithelial dysplasia patients with DNA content abnormality developed high-grade dysplasia or gastric adenocarcinoma within a year and thus may benefit from more thorough and rigorous endoscopic surveillance.
\end{abstract}

Won-Tak Choi

Won-Tak.Choi@ucsf.edu

1 Department of Pathology, University of California at San Francisco, San Francisco, CA 94143, USA

2 Department of Pathology, University of Washington, Seattle, WA 98195, USA

3 Department of Public Health and Preventive Medicine, SUNY Upstate Medical University, Syracuse, NY 13210, USA

4 Department of Pathology, H. Lee Moffitt Cancer Center, Tampa, FL 33612, USA

\section{Introduction}

Gastric epithelial dysplasia represents the penultimate stage of Correa's multi-step gastric carcinogenesis that describes the sequential progression from atrophic gastritis (via Helicobacter pylori infection) to intestinal metaplasia, to dysplasia, and finally to gastric adenocarcinoma [1-5]. While chronic atrophy and intestinal metaplasia are markers of increased risk, gastric epithelial dysplasia represents a direct precursor to gastric adenocarcinoma [4]. The 
prevalence rate of gastric epithelial dysplasia varies worldwide and is higher in East Asia (9-20\%) than in the West $(0.5-4 \%)$ [2, 4-7]. Older males are also at increased risk $[1,8]$ as well as those affected with familial adenomatous polyposis $[9,10]$.

Gastric epithelial dysplasia is morphologically classified into low- and high-grade dysplasia $[1,4,7,11,12]$. The reported progression rate of low-grade dysplasia to a higher neoplastic grade (high-grade dysplasia or gastric adenocarcinoma) varies widely from 0 to $33 \%$ within a mean interval of 10 months to 4 years [2, 4, 7, 13-17], likely representing in part the misclassification of benign reactive mucosal changes as dysplasia. Alternatively, the reported progression of high-grade dysplasia to gastric adenocarcinoma appears higher $(25-85 \%)$ within a mean interval of 4-48 months [2, 4, 7, 13-15, 17-22].

Mass screening has been successfully developed in East Asia with high detection rates of high-grade dysplasia and early gastric adenocarcinoma, leading to improved mortality [23]. Conversely, in the West where there is a lower risk of gastric adenocarcinoma, no surveillance strategy has been regularly implemented with the exception of syndromic patients (and their families). In terms of management, patients with high-grade dysplasia typically undergo endoscopic resection or surgery for a lesion not amenable to endoscopic therapy, followed by a repeat endoscopy within 6 months to a year to screen for synchronous or metachronous gastric adenocarcinoma [4, 7, 24-26]. The management for low-grade dysplasia is not as well defined, but endoscopic resection is standard if the lesion is visible with a repeat endoscopy within a year [4, 7, 24-26]. However, after a diagnosis of low- or high-grade dysplasia, there is no consensus regarding surveillance endoscopy, in particular at what intervals. This is further complicated by the fact that accurate diagnosis and grading of gastric epithelial dysplasia remains challenging with significant interobserver variability among pathologists [2, 27-29].

Adjunct immunohistochemical staining (for p53 and $\beta$ catenin) and molecular marker testing (including $A P C$ and TP53 mutations) have been evaluated, but to date there is no ancillary assay that can reproducibly confirm and/or risk stratify a diagnosis of gastric epithelial dysplasia. For instance, p53 immunostaining, overexpressed in 30-57\% of gastric epithelial dysplasia, can also show strong nuclear staining in 7-23\% of non-dysplastic epithelia [4, 7, 30, 31]. Similarly, no significant association between the incidence of $A P C$ mutations and the grade of dysplasia has been reported [32].

In this regard, we postulated that flow cytometry that measures DNA content abnormality (aneuploidy or elevated $4 \mathrm{~N}$ fraction) can be potentially useful as an adjunct method for the diagnosis and risk assessment of gastric epithelial dysplasia, as we recently demonstrated in Barrett's esophagus [33] and inflammatory bowel disease [34]. However, reports of such analyses on gastric epithelial dysplasia are currently not available, and thus this study examines the utility of DNA content analysis in the diagnosis and risk stratification of gastric epithelial dysplasia. Formalin-fixed paraffin-embedded material was utilized, due to its retrospective availability and its practicality for collection in the clinical setting.

\section{Materials and methods}

\section{Patients and data collection}

A total of 61 dysplastic gastric samples (including 23 highand 38 low-grade dysplasia) from 61 patients diagnosed at the University California at San Francisco Medical Center between 1985 and 2015 were identified using our pathology information system (CoPath). All the dysplastic samples were re-reviewed and confirmed by at least two pathologists (WTC, GYL, and/or KWW) using published criteria [1, 4, $7,11,12]$. Only histologically unequivocal cases of highand low-grade dysplasia were included in the study. Lowgrade dysplasia was defined as having mild to moderate cytologic atypia limited to the basal half of cytoplasm without surface maturation or significant architectural alterations, whereas high-grade dysplasia demonstrated more severe cytologic (including enlarged nuclei, high nuclear to cytoplasmic ratio, prominent nucleoli, loss of polarity, and/or increased mitotic figures) and architectural changes (such as glandular crowding and cribriform formation). Low-grade dysplasia samples included 36 biopsies and 2 surgical resections, whereas high-grade dysplasia samples comprised 19 biopsies and 4 surgical resections. All low- and high-grade dysplasia cases were initially diagnosed on biopsy specimens, with their outcomes determined by reviewing subsequent biopsies and endoscopic/surgical resections as well as clinical notes. When the initial biopsy specimens were not available for flow cytometric analysis (i.e., two surgical resections for lowgrade dysplasia and four surgical resections for high-grade dysplasia), dysplastic samples were obtained from the subsequent surgical resection specimens. We previously demonstrated $100 \%$ concordance in flow cytometric results between prior biopsy and subsequent surgical resection specimens [33]. Twenty-four benign background mucosal samples (without dysplasia, intestinal metaplasia, or Helicobacter pylori) from the same cohort (20 biopsies and 4 surgical resections from 16 low- and 8 high-grade dysplasia cases) were utilized as controls. Hospital electronic medical records were also reviewed to retrieve pertinent demographic (age, gender, and ethnicity), endoscopic appearance (polypoid), and clinicopathologic data (familial 
adenomatous polyposis as well as the presence of Helicobacter pylori infection and intestinal metaplasia). The University California at San Francisco Institutional Review Board for human subjects research approved our study (IRB \# 16-21034).

\section{DNA flow cytometry}

As previously described [33, 34], three to four 60-micron thick sections were cut from each tissue block (without exhausting tissue), and the area of dysplasia was manually dissected from each section to enrich a potential abnormal cell population in a background of normal diploid cells. Each sample was stained with DAPI (4,6-diamidino-2phenylindole; Accurate Chemical \& Scientific Corporation, Westbury, NY) and analyzed with a BD LSRII S854 flow cytometer (BD Biosciences, San Jose, CA) using UV laser excitation. All DNA content histograms were analyzed using the computer program Multicycle (De Novo software, Glendale, CA) based on the published consensus guidelines for clinical DNA flow cytometry (current gold standard to assess DNA content abnormality in the gastrointestinal tract) [35]. DNA aneuploidy was defined as an extra $G_{0} / G_{1}$ peak that was visually distinguishable from the normal DNA diploid $G_{0} / G_{1}$ peak [35]. The presence of $G_{2} /$ tetraploid $(4 \mathrm{~N})$ fraction greater than $6 \%$ (with DNA index of 1.9-2.1) was also considered abnormal due to its strong association with dysplasia or adenocarcinoma in the upper gastrointestinal tract [33]. Two authors (WTC and PSR) interpreted all flow cytometric histograms without knowledge of the histological diagnoses. All the flow cytometric results were validated by the pathologist (PSR) with extensive experience in DNA flow cytometry.

\section{Statistical analysis}

Statistical analysis appropriate for censored data (KaplanMeier curves and Cox proportional hazards model) was utilized, since not all patients reached the endpoint of highgrade dysplasia or gastric adenocarcinoma before follow-up ended. Detection rates at specific time points were calculated from the Kaplan-Meier curves, and a null hypothesis of equal distribution of detection times was assessed using the log-rank test. For 2 low- and 4 high-grade dysplasia cases that did not have initial biopsy material available for flow cytometric analysis, the date when low- or high-grade dysplasia was first diagnosed on biopsy (rather than the date of surgical resection) was used as the starting point. The date of last endoscopy or high-grade dysplasia/gastric adenocarcinoma diagnosis was used to determine the length of follow-up time for outcome analyses. Univariate and/or multivariate Cox proportional hazards models were used to determine the hazard ratios (HRs) for subsequent detection of high-grade dysplasia or gastric adenocarcinoma among different risk factors, including abnormal DNA content, older age, male gender, ethnicity, familial adenomatous polyposis, polypoid endoscopic appearance, Helicobacter pylori infection, and intestinal metaplasia. Both $95 \%$ confidence intervals and $p$-values were calculated using the Asymptotic Wald test. Statistical significance was set at $p<$ 0.05. All analyses were performed using SAS 9.4.

\section{Results}

\section{Clinicopathologic features of gastric epithelial dysplasia}

Table 1 shows the demographic and clinicopathologic characteristics of our cohort. Males (48\%) and females (53\%) were essentially equally represented. The mean age

Table 1 Characteristics of low- and high-grade dysplasia patients identified at the University of California at San Francisco Medical Center between 1985 and 2015

\begin{tabular}{|c|c|c|c|}
\hline & $\begin{array}{l}\text { Entire } \\
\text { cohort }(n \\
=61)\end{array}$ & $\begin{array}{l}\text { Low-grade } \\
\text { dysplasia }(n= \\
38)\end{array}$ & $\begin{array}{l}\text { High-grade } \\
\text { dysplasia }(n= \\
23)\end{array}$ \\
\hline Mean age, years (range) & $63(9-92)$ & $58(9-87)$ & $73(31-92)$ \\
\hline \multicolumn{4}{|l|}{ Gender } \\
\hline Male & $29(48 \%)$ & $19(50 \%)$ & $10(44 \%)$ \\
\hline Female & $32(53 \%)$ & $19(50 \%)$ & $13(57 \%)$ \\
\hline \multicolumn{4}{|l|}{ Ethnicity } \\
\hline Caucasians & $22(36 \%)$ & $15(40 \%)$ & $7(30 \%)$ \\
\hline Non-Caucasians & $39(64 \%)$ & $23(61 \%)$ & $16(70 \%)$ \\
\hline Asians & $21(34 \%)$ & $12(32 \%)$ & $9(39 \%)$ \\
\hline Hispanics & $8(13 \%)$ & $6(16 \%)$ & $2(9 \%)$ \\
\hline African Americans & $5(8 \%)$ & $3(8 \%)$ & $2(9 \%)$ \\
\hline Others & $5(8 \%)$ & $2(5 \%)$ & $3(13 \%)$ \\
\hline $\begin{array}{l}\text { Familial adenomatous } \\
\text { polyposis }\end{array}$ & $14(23 \%)$ & $12(32 \%)$ & $2(9 \%)$ \\
\hline Polypoid appearance & $49(80 \%)$ & $31(82 \%)$ & $18(78 \%)$ \\
\hline Helicobacter pylori & $10(16 \%)$ & $7(18 \%)$ & $3(13 \%)$ \\
\hline Intestinal metaplasia & $39(64 \%)$ & $24(63 \%)$ & $15(65 \%)$ \\
\hline \multicolumn{4}{|l|}{ Location } \\
\hline Antrum & $26(43 \%)$ & $16(42 \%)$ & $10(44 \%)$ \\
\hline Fundus & $12(20 \%)$ & $7(18 \%)$ & $5(22 \%)$ \\
\hline Angularis & $7(12 \%)$ & $2(5 \%)$ & $5(22 \%)$ \\
\hline Cardia & $6(10 \%)$ & $5(13 \%)$ & $1(4 \%)$ \\
\hline Pylorus & $2(3 \%)$ & $1(3 \%)$ & $1(4 \%)$ \\
\hline Anastomosis & $1(2 \%)$ & $1(3 \%)$ & $0(0 \%)$ \\
\hline Unknown & $7(12 \%)$ & $6(16 \%)$ & $1(4 \%)$ \\
\hline DNA content abnormality & $23(38 \%)$ & $5(13 \%)$ & $18(78 \%)$ \\
\hline $\begin{array}{l}\text { Mean follow-up time, } \\
\text { months (range) }\end{array}$ & $20(0-147)$ & $24(0-147)$ & $13(0-109)$ \\
\hline
\end{tabular}



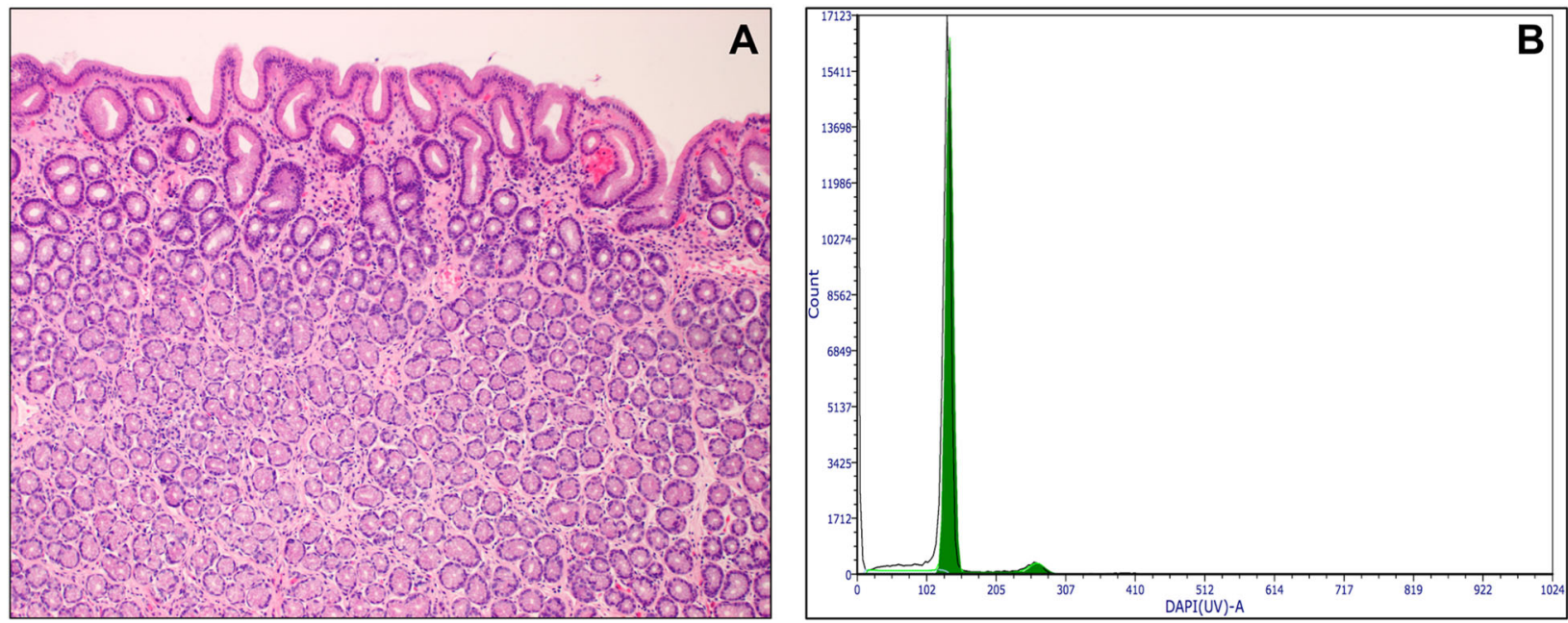

Fig. 1 a Normal antral-type gastric mucosa without dysplasia. b Only normal diploid population (green) is present in this DNA histogram

of all gastric epithelial dysplasia patients was 63 years, but high-grade dysplasia patients (mean 73 years) were older than low-grade dysplasia patients (mean 58 years). As previously demonstrated [2, 4-7], gastric epithelial dysplasia was more common in non-Caucasian patients (64\%) than Caucasian counterpart (36\%). Gastric epithelial dysplasia was detected throughout the stomach but most frequently involved the antrum (43\%), followed by fundus $(20 \%)$, angularis $(12 \%)$, cardia $(10 \%)$, pylorus $(3 \%)$, and anastomotic site $(2 \%)$. The specific location was unknown in seven instances (12\%). Gastric epithelial dysplasia usually presented as a polyp/nodule ( $80 \%)$ but also as a flat lesion or detected incidentally in a normal appearing mucosa (20\%). Fourteen cases of gastric epithelial dysplasia (23\%) developed in the setting of familial adenomatous polyposis. Helicobacter pylori infection and intestinal metaplasia were common in gastric epithelial dysplasia patients, as they were present in 16 and $64 \%$ of cases, respectively.

\section{DNA content analysis in normal gastric mucosa}

No abnormal DNA content was identified in the 24 nondysplastic samples (Fig. 1).

\section{DNA content abnormality in low-grade dysplasia}

Five (13\%; all biopsies) of 38 low-grade dysplasia samples demonstrated DNA content abnormality (Fig. 2; Table 1). Four samples had aneuploidy, whereas the remaining 1 sample showed elevated $4 \mathrm{~N}$ fraction. The finding of abnormal DNA content at baseline low-grade dysplasia was a significant risk factor for subsequent detection of highgrade dysplasia or gastric adenocarcinoma with the estimated univariate and multivariate HRs of $6.9(p=0.001)$ and $5.9(p=0.005)$, respectively (Table 2). All five lowgrade dysplasia patients $(100 \%)$ with abnormal DNA content were found to have high-grade dysplasia (3 patients) or gastric adenocarcinoma ( 2 patients) within a mean followup time of 10 months. More precisely, 1 and 4-year detection rates of high-grade dysplasia or gastric adenocarcinoma in these patients were $80 \%(p=0.003,95 \%$ confidence interval $=[19-96 \%])$ and $100 \%(p=0.005$, $95 \%$ confidence interval $=[15-100 \%]$, respectively (Fig. 2c). Three patients developed high-grade dysplasia (2 patients) or gastric adenocarcinoma (1 patient) at an adjacent or distant segment of stomach from the initial biopsy, whereas the remaining 2 patients were subsequently found to have high-grade dysplasia (1 patient) or gastric adenocarcinoma (1 patient) at the prior biopsy site. By contrast, of 33 low-grade dysplasia patients with normal DNA content, 10 patients (30\%) developed high-grade dysplasia (5 patients) or gastric adenocarcinoma (5 patients) within a mean follow-up time of 26 months. Five patients were diagnosed with high-grade dysplasia (3 patients) or gastric adenocarcinoma (2 patients) at the site of prior biopsy, while the remaining 5 patients developed high-grade dysplasia (2 patients) or gastric adenocarcinoma (3 patient) at an adjacent or distant segment of stomach from the initial biopsy. 1, 4, and 12-year detection rates of a higher grade lesion (high-grade dysplasia or gastric adenocarcinoma) in these patients were 23,32 , and $54 \%$, respectively.

Analyzing high-grade dysplasia and gastric adenocarcinoma as separate outcomes, 7 (18\%) of 38 low-grade dysplasia patients developed gastric adenocarcinoma (4 patients at a different site and 3 patients at the prior biopsy site) within a mean follow-up time of 14 months, whereas 8 $(21 \%)$ patients were diagnosed with high-grade dysplasia only ( 4 patients at a different site and 4 patients at the prior biopsy site) within a mean follow-up time of 13 months. 

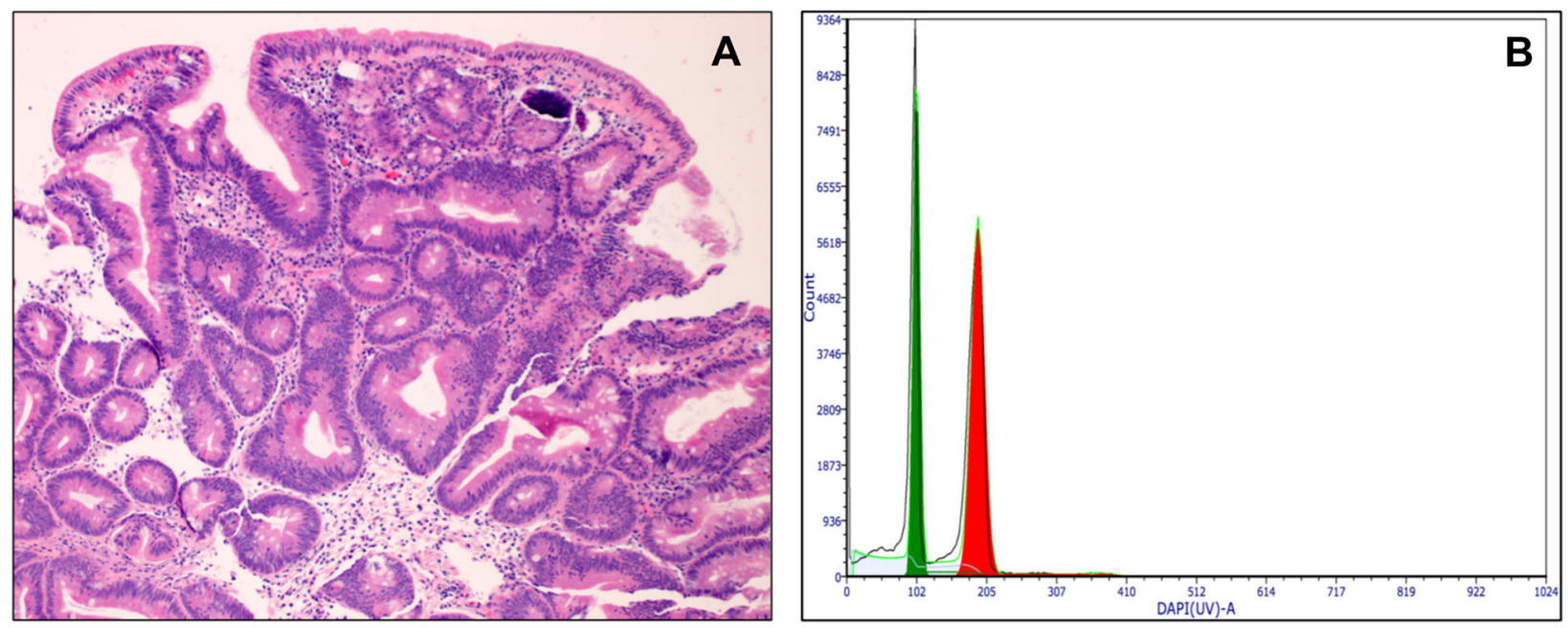

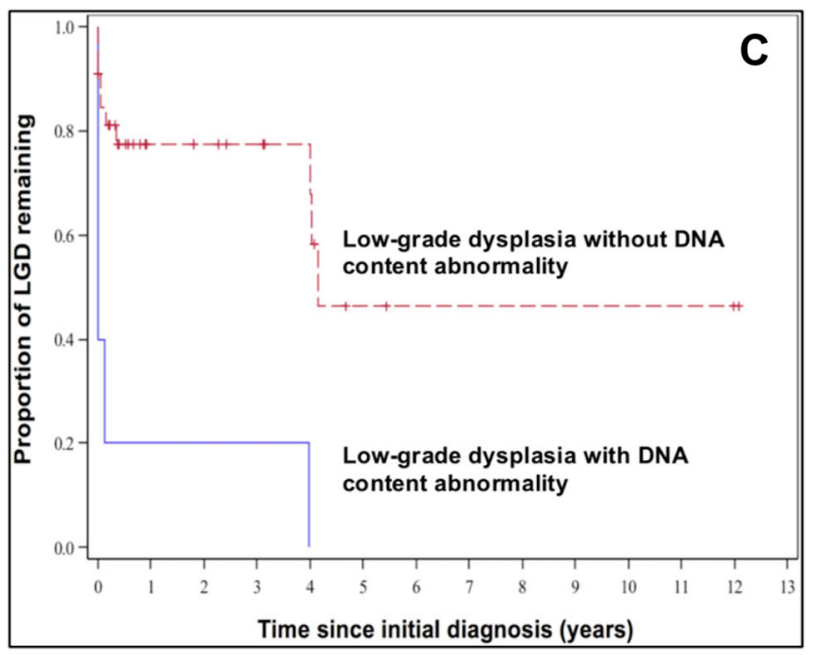

Fig. 2 a Case of low-grade dysplasia characterized by mild to moderate cytologic atypia without surface maturation or significant architectural disarray. b DNA histogram shows a distinct aneuploid population (red) in addition to a normal diploid population (green). c Detection of high-grade dysplasia or gastric adenocarcinoma in lowgrade dysplasia patients with abnormal DNA content at baseline. 1 and 4 -year detection rates of high-grade dysplasia or gastric adenocarcinoma in low-grade dysplasia patients with DNA content abnormality were $80 \%(p=0.003)$ and $100 \%(p=0.005)$, respectively, whereas

The patients who developed gastric adenocarcinoma were slightly older (mean 75 years) and all men, whereas those diagnosed with high-grade dysplasia only included 3 males (38\%) and 5 females $(63 \%)$ with a mean age of 70 years. The overall 1, 4, 5, and 12-year detection rates of highgrade dysplasia or gastric adenocarcinoma in all low-grade dysplasia patients (regardless of flow cytometric results) were $30,46,63$, and $63 \%$, respectively (95\% confidence intervals $=[15-45 \%],[23-68 \%],[38-88 \%]$, and $[38-88 \%]$, respectively) (Fig. 2d).

Since older age $[2-4,8,26,36]$, male gender $[1,2,4,8]$, non-Caucasian populations [4, 6, 22], familial adenomatous polyposis $[4,9,10]$, polypoid endoscopic appearance $[4$,

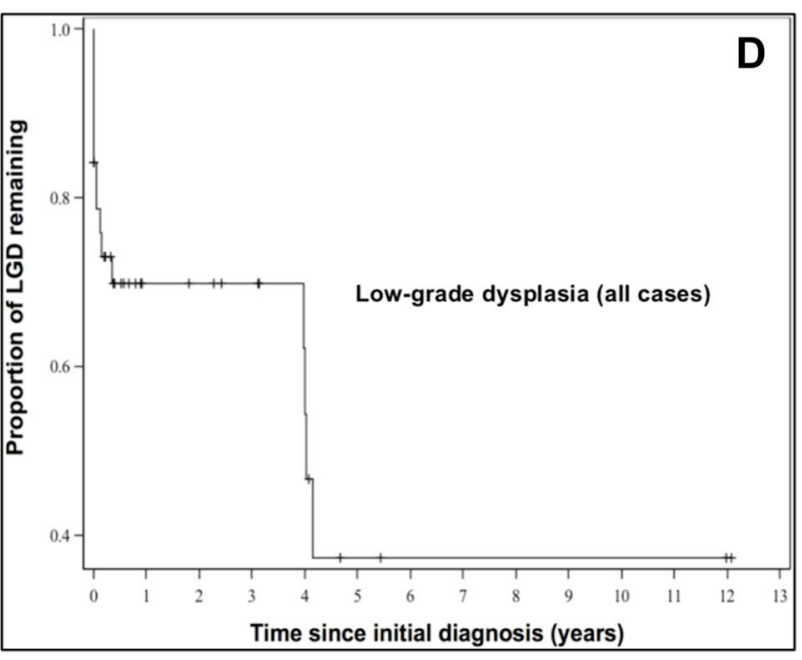

$23 \%$ of low-grade dysplasia cases in the setting of normal DNA content were found to have high-grade dysplasia or gastric adenocarcinoma within 1 year, with 32 and 54\% detected within 4 and 12 years, respectively. Each tick represents a patient person being censored. d Overall detection of high-grade dysplasia or gastric adenocarcinoma in low-grade dysplasia patients regardless of flow cytometric results. 1, 4, 5, and 12-year detection rates were $30,46,63$, and $63 \%$, respectively. Each tick represents a patient being censored

37], Helicobacter pylori infection [4, 5, 38], and intestinal metaplasia [3-5, 22, 26, 36, 39, 40] have been reported as significant risk factors for the development of gastric epithelial dysplasia or gastric adenocarcinoma, they were analyzed on univariate and multivariate Cox proportional hazards models. On univariate analysis, abnormal DNA content $(\mathrm{HR}=6.9, p=0.001)$, older age $(\mathrm{HR}=1.1, p=$ 0.005 ), and familial adenomatous polyposis ( $\mathrm{HR}=9.7, p=$ 0.029 ) were significantly associated with subsequent detection of high-grade dysplasia or gastric adenocarcinoma (Table 2). However, only abnormal DNA content maintained a significantly elevated HR of $5.9(p=0.005)$ in the multivariate Cox model. Although statistically significant, 
the HR of older age was only minimally elevated $(\mathrm{HR}=$ $1.1, p=0.013)$. The presence of Helicobacter pylori $(\mathrm{HR}=$ $0.4, p=0.148)$, intestinal metaplasia $(\mathrm{HR}=0.4, p=0.166)$, and polypoid endoscopic appearance $(\mathrm{HR}=1.3, p=0.684)$ were not significantly associated with an increased risk for subsequent detection of high-grade dysplasia or gastric adenocarcinoma. Similarly, male gender $(\mathrm{HR}=0.4, p=$ $0.124)$ and ethnicity $(\mathrm{HR}=1.3, p=0.602)$ were not significant risk factors for subsequent detection of high-grade dysplasia or gastric adenocarcinoma.

Table 2 Univariate and multivariate Cox proportional hazards models with high-grade dysplasia or gastric adenocarcinoma as the outcome in low-grade dysplasia patients

\begin{tabular}{llll}
\hline & \multicolumn{2}{l}{ Outcome of low-grade dysplasia } \\
\cline { 2 - 4 } & $\begin{array}{l}\text { Detection of high-grade dysplasia or gastric } \\
\text { adenocarcinoma }\end{array}$ \\
\cline { 2 - 4 } $\begin{array}{l}\text { Univariate Cox model } \\
\text {-Value }\end{array}$ & $\begin{array}{l}\text { Hazard } \\
\text { ratio }\end{array}$ & $\begin{array}{l}95 \% \text { confidence } \\
\text { interval }\end{array}$ \\
\hline $\begin{array}{l}\text { DNA content } \\
\text { abnormality }\end{array}$ & 0.001 & 6.9 & $2.130-22.468$ \\
$\begin{array}{l}\text { Older age } \\
\text { Male gender }\end{array}$ & 0.005 & 1.1 & $1.017-1.103$ \\
$\begin{array}{l}\text { Non-Caucasian } \\
\text { Familial adenomatous } \\
\text { polyposis }\end{array}$ & 0.124 & 0.4 & $0.146-1.262$ \\
$\begin{array}{l}\text { Polypoid appearance } \\
\text { Helicobacter pylori }\end{array}$ & 0.684 & 1.3 & $0.459-3.837$ \\
$\begin{array}{l}\text { Intestinal metaplasia } \\
\text { Multivariate Cox model }\end{array}$ & 0.148 & 0.7 & $1.265-74.924$ \\
$\begin{array}{l}\text { DNA content } \\
\text { abnormality }\end{array}$ & 0.005 & 0.4 & $0.366-4.631$ \\
$\begin{array}{l}\text { Familial adenomatous } \\
\text { polyposis }\end{array}$ & 0.485 & 0.4 & $0.123-1.370$ \\
\begin{tabular}{l} 
Age \\
\hline
\end{tabular} & 0.013 & 1.1 & $0.115-1.451$ \\
\hline
\end{tabular}

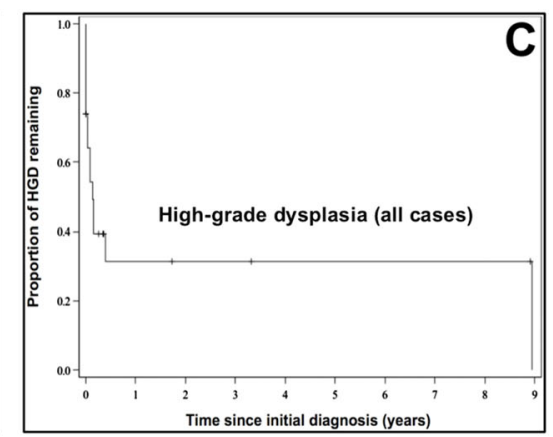

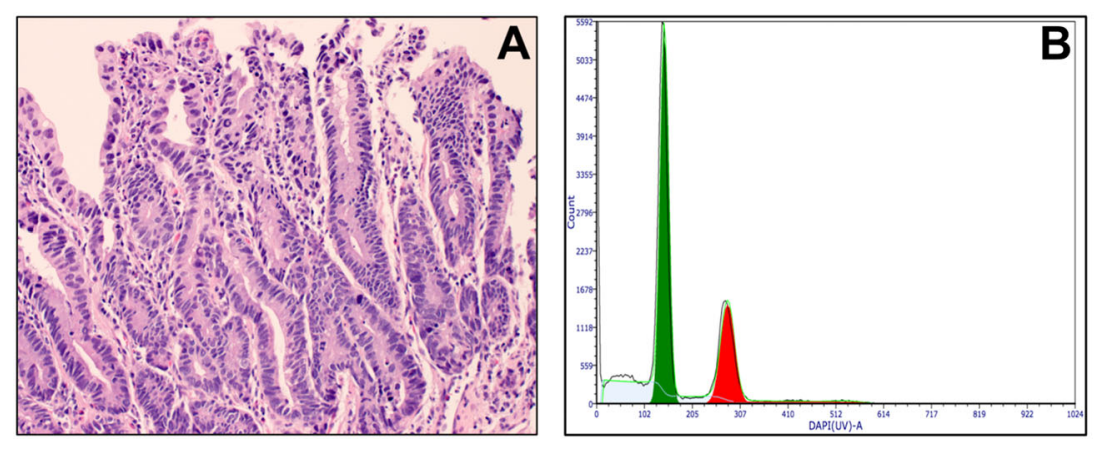

Fig. 3 a Case of high-grade dysplasia characterized by marked cytologic atypia and architectural abnormalities (such as glandular crowding). b DNA histogram shows a discrete aneuploid peak (red) that is distinct from a normal diploid population (green). c Overall
DNA content abnormality in high-grade dysplasia

Overall, $15(65 \%)$ of 23 high-grade dysplasia patients developed gastric adenocarcinoma. Thirteen patients were diagnosed with gastric adenocarcinoma at the site of prior biopsy, whereas the remaining two patients developed metastatic cancer. The remaining 8 patients subsequently underwent endoscopic or surgical resection, and there was no evidence of gastric adenocarcinoma within a mean follow-up time of 23 months. The patients with gastric adenocarcinoma included 7 males and 8 females with a mean age of 70 years, whereas those without gastric adenocarcinoma included 3 males and 5 females with a mean age of 79 years. The mean time to subsequent detection of gastric adenocarcinoma was 9 months. The overall detection rates of gastric adenocarcinoma in all high-grade dysplasia patients (regardless of flow cytometric results) were 46, 61, 69, and $100 \%$ within 1 month, 2.5 months, 6 months, and 9 years, respectively (95\% confidence intervals $=[25-67 \%],[40-82 \%],[47-90 \%]$, and [100-100\%], respectively) (Fig. 3c).

DNA content abnormality was detected in 18 (78\%) of 23 high-grade dysplasia samples (Fig. 3; Table 1). Twelve cases demonstrated aneuploidy, whereas the remaining 6 samples showed elevated $4 \mathrm{~N}$ fraction. Thirteen (72\%) of the 18 high-grade dysplasia cases were subsequently found to have gastric adenocarcinoma (11 cases at the prior biopsy site and 2 cases as metastatic cancer), whereas only $2(40 \%)$ of 5 high-grade dysplasia cases with normal DNA content developed gastric adenocarcinoma at the site of prior biopsy $(\mathrm{HR}=2.5)$. However, this difference was not statistically significant $(p=0.226)$ (Table 3). Similarly, older age (HR $=1.0, p=0.923)$, male gender $(\mathrm{HR}=0.5, p=0.199)$, ethnicity $(\mathrm{HR}=1.4, \quad p=0.526)$, familial adenomatous polyposis $(\mathrm{HR}=0.9, p=0.946)$, polypoid endoscopic appearance $(\mathrm{HR}=1.0, p=0.962)$, Helicobacter pylori infection $(\mathrm{HR}=1.1, p=0.885)$, and intestinal metaplasia

detection of gastric adenocarcinoma in high-grade dysplasia patients regardless of flow cytometric results. The detection rates were 46,61 , 69, and 100\% within 1 month, 2.5 months, 6 months, and 9 years, respectively. Each tick represents a patient being censored 
Table 3 Univariate Cox proportional hazards model with gastric adenocarcinoma as the outcome in high-grade dysplasia patients

\begin{tabular}{llll}
\hline & \multicolumn{2}{l}{ Outcome of high-grade dysplasia } \\
\cline { 2 - 4 } & \multicolumn{2}{c}{ Detection of gastric adenocarcinoma } \\
\cline { 2 - 4 } Univariate Cox model & $p$-Value & $\begin{array}{l}\text { Hazard } \\
\text { ratio }\end{array}$ & $\begin{array}{l}95 \% \text { confidence } \\
\text { interval }\end{array}$ \\
\hline $\begin{array}{l}\text { DNA content } \\
\text { abnormality }\end{array}$ & 0.226 & 2.5 & $0.561-11.542$ \\
Older age & 0.923 & 1.0 & $0.964-1.034$ \\
Male gender & 0.199 & 0.5 & $0.169-1.446$ \\
Non-Caucasian & 0.526 & 1.4 & $0.475-4.287$ \\
$\begin{array}{l}\text { Familial adenomatous } \\
\text { polyposis }\end{array}$ & 0.946 & 0.9 & $0.209-4.306$ \\
Polypoid appearance & 0.962 & 1.0 & $0.286-3.719$ \\
Helicobacter pylori & 0.885 & 1.1 & $0.249-5.019$ \\
Intestinal metaplasia & 0.083 & 2.5 & $0.884-7.347$ \\
\hline
\end{tabular}

$(\mathrm{HR}=2.5, p=0.083)$ were not significantly associated with subsequent detection of gastric adenocarcinoma.

\section{Discussion}

Although the development of optimal surveillance strategies for gastric epithelial dysplasia remains challenging, especially in the West, the detection of high-grade dysplasia is an indication for at least endoscopic resection (if not surgery depending on the characteristics of the case) [24-26]. It is therefore critical that the diagnosis of such lesions be confirmed and its consequences fully appreciated. In this regard, the majority of high-grade dysplasia cases (up to $78 \%$ in our series) showed DNA content abnormality, while all of the control samples demonstrated normal DNA content, suggesting that DNA flow cytometry can provide confirmatory evidence to a morphologic impression or suspicion of high-grade dysplasia in challenging cases, as well as in situations where there is discordant interpretation among expert gastrointestinal pathologists. This is crucial, since there is currently no ancillary test, either immunohistochemical or molecular, that can reproducibly diagnose gastric epithelial dysplasia. For instance, p53 staining is detected in $30-57 \%$ of gastric epithelial dysplasia but also in $7-23 \%$ of non-dysplastic samples $[4,7,30,31]$. Similarly, abnormal $\beta$-catenin nuclear staining is observed in only $38 \%$ of gastric epithelial dysplasia cases [41]. Furthermore, the high frequency of DNA content abnormality in high-grade dysplasia supports the finding that gastric adenocarcinoma with chromosomal instability (CIN) represents the largest subtype of gastric cancer [42, 43]. The high-grade dysplasia cases with normal DNA content likely represent the subsets of gastric adenocarcinoma that develop via an alternative pathway. Indeed, a recent molecular classification of gastric adenocarcinomas described three additional molecular subtypes which typically do not result in DNA content abnormality, including (1) EpsteinBarr virus (EBV)-positive tumors which display DNA hypermethylation; (2) microsatellite instable tumors; and (3) genomically stable tumors [42, 43].

The overall detection rates of gastric adenocarcinoma in all high-grade dysplasia patients (regardless of flow cytometric results) were 46, 61, 69, and $100 \%$ within 1 month, 2.5 months, 6 months, and 9 years, respectively, compatible with the previously reported progression rate of high-grade dysplasia ranging from 25 to $85 \%$ [2, 4, 7, 13-15, 17-22]. While DNA content abnormality in the setting of highgrade dysplasia was associated with the estimated HR of 2.5 for subsequent detection of gastric adenocarcinoma, this finding did not achieve statistical significance in the present sample size $(p=0.226)$ (Table 3$)$. Nevertheless, the findings still highlight the importance of complete resection of high-grade dysplasia followed by close endoscopic surveillance (every 3 months within the first year, followed by a long-term, annual endoscopy), given the very high risk for gastric adenocarcinoma.

The issue regarding low-grade dysplasia is more unsettled. It is currently unknown if a CIN mechanism with DNA content abnormality takes part in the progression of low- to high-grade dysplasia or gastric adenocarcinoma. In this regard, our data show that abnormal DNA content in lowgrade dysplasia is significantly associated with subsequent detection of high-grade dysplasia or gastric adenocarcinoma with the estimated univariate and multivariate HRs of 6.9 $(p=0.001)$ and $5.9(p=0.005)$, respectively (Table 2$)$. In fact, the majority of low-grade dysplasia patients $(80 \%)$ with abnormal DNA content were found to have high-grade dysplasia or gastric adenocarcinoma within a year with $100 \%$ detected within 4 years, whereas patients with lowgrade dysplasia but with normal DNA content had 1,4 , and 12 -year detection rates of 23,32 , and $54 \%$, respectively (Fig. 2c). This is consistent with the previous report that patients who develop gastric adenocarcinoma via an alternative pathway other than the CIN pathway (in particular, EBV-positive and microsatellite instable subgroups) have superior survival outcomes [43]. Of note, we recently reported a similar association of DNA content abnormality and low-grade dysplasia with later development of highgrade dysplasia or cancer in Barrett's esophagus [33] and inflammatory bowel disease [34]. Considering that there is currently no optimal surveillance strategy available after a diagnosis of low-grade dysplasia, our results suggest that low-grade dysplasia patients, especially those with abnormal DNA content, should benefit from more enhanced endoscopic surveillance than those with normal DNA content, especially during the first year (to exclude synchronous 
or rapidly developing high-grade dysplasia or gastric adenocarcinoma), followed by ongoing annual endoscopy (at least for the next few years). However, we note that 1, 4, and 12-year detection rates of high-grade dysplasia or gastric adenocarcinoma in low-grade dysplasia patients with normal DNA content were 23,32 , and $54 \%$, respectively (Fig. 2c), which is still consistent with the previously reported progression rate of low-grade dysplasia ranging from 0 to $33 \%$ [2, 4, 7, 13-17]. As such, a negative flow cytometric result after an initial low-grade dysplasia diagnosis may not offer complete assurance of safety, and endoscopic resection followed by a long-term, possibly annual endoscopy (in conjunction with flow cytometry) may be appropriate for these patients.

A few earlier studies demonstrated a correlation between the frequency of DNA content abnormality and increasing histological grade of gastric epithelial dysplasia using either formalin-fixed paraffin-embedded [44, 45] or fresh tissue [46]. However, none of these studies identified DNA content abnormality in low-grade dysplasia or correlated histologic grade of dysplasia with its outcome. For instance, Macartney et al. reported that none of 17 lowgrade dysplasia and 5 of 7 high-grade dysplasia cases showed DNA content abnormality [44], whereas Yasa et al. demonstrated that none of 6 low-grade dysplasia and 3 of 6 high-grade dysplasia cases showed DNA content abnormality [46]. Similarly, Tanaka et al. reported that all 15 lowgrade dysplasia cases showed normal DNA content using static cytofluorometry [45]. In our series, 5 (13\%) of 38 low-grade dysplasia and $18(78 \%)$ of 23 high-grade dysplasia samples demonstrated DNA content abnormality, and all 5 low-grade dysplasia patients with abnormal DNA content developed high-grade dysplasia or gastric adenocarcinoma. These results seem to indicate that large-scale chromosomal changes do occur in a subset of low-grade dysplasia cases, and that the presence of DNA content abnormality detected at baseline low-grade dysplasia can potentially serve as a biomarker for malignant potential. This is further supported by the previous finding that DNA copy number changes (including gains of chromosomes $11 \mathrm{q}, 9 \mathrm{q}$, and 8 as well as losses of chromosomes 5q, 6, 10, and 13) are common in low-grade dysplasia [47]. We also note that the frequency of DNA content abnormality in our high-grade dysplasia cohort is higher than previously reported. This is possibly due to the fact that these earlier studies failed to macroscopically dissect dysplastic epithelial cells from background normal cells [44, 46], leading to an increase in the proportion of non-dysplastic epithelial cells and thus, reducing the sensitivity to detect dysplastic epithelial cells with abnormal DNA content (dilutional effects).

Although intestinal metaplasia is considered a premalignant condition for gastric adenocarcinoma [36, 39, 40] and often associated with Helicobacter pylori infection [48], neither intestinal metaplasia nor Helicobacter pylori was found to be an important risk factor for subsequent detection of high-grade dysplasia or gastric adenocarcinoma in our cohort. Also despite the fact that $23 \%$ of gastric epithelial dysplasia in our cohort developed in the setting of familial adenomatous polyposis, there was no significant association between familial adenomatous polyposis and subsequent detection of high-grade dysplasia or gastric adenocarcinoma based on our multivariate analysis, which is consistent with the previous finding that the risk for highgrade dysplasia or gastric adenocarcinoma is not significantly increased in Western patients with familial adenomatous polyposis [9, 10, 49].

One possible limitation of our study is that all the patients in our cohort were referred to or seen at the University California at San Francisco Medical Center and may differ from those seen at community practices where screening is performed. Also because the study was designed to identify risk factors predictive of subsequent detection of high-grade dysplasia or gastric adenocarcinoma occurring within 12 years of initial low- or high-grade dysplasia biopsy, we cannot completely exclude the possibility that some of these demographic (older age, male gender, and ethnicity), endoscopic (polypoid), and clinicopathologic risk factors (familial adenomatous polyposis, Helicobacter pylori infection, and intestinal metaplasia) may be associated with an increased risk for subsequent detection of high-grade dysplasia or gastric adenocarcinoma with a longer follow-up time.

In conclusion, in low and average risk populations, a combination of confirmed low-grade dysplasia diagnosis and DNA content abnormality can identify a subset of lowgrade dysplasia patients who are at increased risk for developing high-grade dysplasia or gastric adenocarcinoma and thus may benefit from enhanced endoscopic surveillance. The presence of DNA content abnormality can also provide confirmatory evidence to a morphologic suspicion of high-grade dysplasia.

Funding University California at San Francisco Department of Pathology

\section{Compliance with ethical standards}

Conflict of interest The authors declare that they have no conflict of interest.

\section{References}

1. Setia N, Lauwers GY. Gastric dysplasia: update and practical approach. Diagn Histopathol. 2015;21:312-22.

2. Lauwers GY, Riddell RH. Gastric epithelial dysplasia. Gut. 1999;45:784-90. 
3. Correa P, Haenszel W, Cuello C, et al. Gastric precancerous process in a high risk population: cohort follow-up. Cancer Res. 1990;50:4737-40.

4. Lauwers GY, Srivastava A. Gastric preneoplastic lesions and epithelial dysplasia. Gastroenterol Clin North Am. 2007;36:813-29.

5. Yakirevich E, Resnick MB. Pathology of gastric cancer and its precursor lesions. Gastroenterol Clin North Am. 2013;42:261-84.

6. Choi CE, Sonnenberg A, Turner K, et al. High prevalence of gastric preneoplastic lesions in East Asians and Hispanics in the USA. Dig Dis Sci. 2015;60:2070-6.

7. Srivastava A, Lauwers GY. Gastric epithelial dysplasia: the Western perspective. Dig Liver Dis. 2008;40:641-9.

8. Raftopoulos SC, Kumarasinghe P, de Boer B, et al. Gastric intraepithelial neoplasia in a Western population. Eur J Gastroenterol Hepatol. 2012;24:48-54.

9. Ngamruengphong S, Boardman LA, Heigh RI, et al. Gastric adenomas in familial adenomatous polyposis are common, but subtle, and have a benign course. Hered Cancer Clin Pract. 2014; $12: 4$.

10. Wood LD, Salaria SN, Cruise MW, et al. Upper GI tract lesions in familial adenomatous polyposis (FAP): enrichment of pyloric gland adenomas and other gastric and duodenal neoplasms. Am J Surg Pathol. 2014;38:389-93.

11. Schlemper RJ, Riddell RH, Kato Y, et al. The Vienna classification of gastrointestinal epithelial neoplasia. Gut. 2000;47:251-5.

12. Goldstein NS, Lewin KJ. Gastric epithelial dysplasia and adenoma: historical review and histological criteria for grading. Hum Pathol. 1997;28:127-33.

13. Kokkola A, Haapiainen R, Laxén F, et al. Risk of gastric carcinoma in patients with mucosal dysplasia associated with atrophic gastritis: a follow up study. J Clin Pathol. 1996;49:979-84.

14. Bearzi I, Brancorsini D, Santinelli A, et al. Gastric dysplasia: a ten-year follow-up study. Pathol Res Pract. 1994;190:61-8.

15. Rugge M, Farinati F, Baffa R, et al. Gastric epithelial dysplasia in the natural history of gastric cancer: a multicenter prospective follow-up study. Interdisciplinary Group on Gastric Epithelial Dysplasia. Gastroenterology. 1994;107:1288-96.

16. Park SY, Jeon SW, Jung MK, et al. Long-term follow-up study of gastric intraepithelial neoplasias: progression from low-grade dysplasia to invasive carcinoma. Eur J Gastroenterol Hepatol. 2008;20:966-70.

17. Rugge M, Farinati F, Di Mario F, et al. Gastric epithelial dysplasia: a prospective multicenter follow-up study from the Interdisciplinary Group on Gastric Epithelial Dysplasia. Hum Pathol. 1991;22:1002-8.

18. Kolodziejczyk P, Yao T, Oya M, et al. Long-term follow-up study of patients with gastric adenomas with malignant transformation. An immunohistochemical and histochemical analysis. Cancer. 1994;74:2896-907.

19. de Vries AC, van Grieken NC, Looman CW, et al. Gastric cancer risk in patients with premalignant gastric lesions: a nationwide cohort study in the Netherlands. Gastroenterology. 2008;134:945-52.

20. Lansdown M, Quirke P, Dixon MF, et al. High grade dysplasia of the gastric mucosa: a marker for gastric carcinoma. Gut. 1990;31:977-83.

21. Farinati F, Rugge M, Di Mario F, et al. Early and advanced gastric cancer in the follow-up of moderate and severe gastric dysplasia patients. A prospective study. I.G.G.E.D.-Interdisciplinary Group on Gastric Epithelial Dysplasia. Endoscopy. 1993;25:261-4.

22. Li D, Bautista MC, Jiang SF, et al. Risks and predictors of gastric adenocarcinoma in patients with gastric intestinal metaplasia and dysplasia: a population-based study. Am J Gastroenterol. 2016;111:1104-13.
23. Hamashima C, Shibuya D, Yamazaki H, et al. The Japanese guidelines for gastric cancer screening. Jpn J Clin Oncol. 2008;38:259-67.

24. ASGE Standards of Practice Committee, Evans JA, Chandrasekhara $\mathrm{V}$, et al. The role of endoscopy in the management of premalignant and malignant conditions of the stomach. Gastrointest Endosc. 2015;82:1-8.

25. Shaib YH, Rugge M, Graham DY, et al. Management of gastric polyps: an endoscopy-based approach. Clin Gastroenterol Hepatol. 2013;11:1374-84.

26. Goddard AF, Badreldin R, Pritchard DM, et al. The management of gastric polyps. Gut. 2010;59:1270-6.

27. Fertitta AM, Comin U, Terruzzi V, et al. Clinical significance of gastric dysplasia: a multicenter follow-up study. Gastrointestinal Endoscopic Pathology Study Group. Endoscopy. 1993;25:265-8.

28. de Dombal FT, Price AB, Thompson H, et al. The British Society of Gastroenterology early gastric cancer/dysplasia survey: an interim report. Gut. 1990;31:115-20.

29. Sarela AI, Scott N, Verbeke CS, et al. Diagnostic variation and outcome for high-grade gastric epithelial dysplasia. Arch Surg. 2005;140:644-9.

30. Dong B, Xie YQ, Chen K, et al. Differences in biological features of gastric dysplasia, indefinite dysplasia, reactive hyperplasia and discriminant analysis of these lesions. World J Gastroenterol. 2005;11:3595-600.

31. Teh M, Tan KB, Seet BL, et al. Study of p53 immunostaining in the gastric epithelium of cagA-positive and cagA-negative Helicobacter pylori gastritis. Cancer. 2002;95:499-505.

32. Tamura G, Maesawa C, Suzuki Y, et al. Mutations of the APC gene occur during early stages of gastric adenoma development. Cancer Res. 1994;54:1149-51.

33. Choi WT, Tsai JH, Rabinovitch PS, et al. Diagnosis and risk stratification of Barrett's dysplasia by flow cytometric DNA analysis of paraffin-embedded tissue. Gut 2017. https://doi.org/10. 1136/gutjnl-2017-313815

34. Tsai JH, Rabinovitch PS, Huang D, et al. Association of aneuploidy and flat dysplasia with development of high-grade dysplasia or colorectal cancer in patients with inflammatory bowel disease. Gastroenterology. 2017;153:1492-95.

35. Shankey TV, Rabinovitch PS, Bagwell B, et al. Guidelines for implementation of clinical DNA cytometry. International Society for Analytical Cytology. Cytometry. 1993;14:472-7.

36. Vannella L, Lahner E, Osborn J, et al. Risk factors for progression to gastric neoplastic lesions in patients with atrophic gastritis. Aliment Pharmacol Ther. 2010;31:1042-50.

37. Zhao G, Xue M, Hu Y, et al. How commonly is the diagnosis of gastric low grade dysplasia upgraded following endoscopic resection? A meta-analysis. PLoS ONE. 2015;10:e0132699.

38. You WC, Zhang L, Gail MH, et al. Gastric dysplasia and gastric cancer: Helicobacter pylori, serum vitamin $\mathrm{C}$, and other risk factors. J Natl Cancer Inst. 2000;92:1607-12.

39. den Hoed CM, Holster IL, Capelle LG, et al. Follow-up of premalignant lesions in patients at risk for progression to gastric cancer. Endoscopy. 2013;45:249-56.

40. Whiting JL, Sigurdsson A, Rowlands DC, et al. The long term results of endoscopic surveillance of premalignant gastric lesions. Gut. 2002;50:378-81.

41. Smith GV, Feakins R, Farthing MJ, et al. Cyclooxygenase 2, p53, beta-catenin, and APC protein expression in gastric adenomatous polyps. Am J Clin Pathol. 2005;123:415-20.

42. Cancer Genome Atlas Research Network. Comprehensive molecular characterization of gastric adenocarcinoma. Nature. 2014;513:202-9.

43. Setia N, Agoston AT, Han HS, et al. A protein and mRNA expression-based classification of gastric cancer. Mod Pathol. 2016;29:772-84. 
44. Macartney JC, Camplejohn RS. DNA flow cytometry of histological material from dysplastic lesions of human gastric mucosa. $\mathbf{J}$ Pathol. 1986;150:113-8.

45. Tanaka A, Takemura-Tsukashita S, Kushima R, et al. Low-grade gastric adenomas/dysplasias: phenotypic expression, DNA ploidy pattern, and $\mathrm{LOH}$ at microsatellites linked to the APC gene. Pathol Res Pract. 2008;204:1-9.

46. Yasa MH, Bektas A, Yukselen V, et al. DNA analysis and DNA ploidy in gastric cancer and gastric precancerous lesions. Int J Clin Pract. 2005;59:1029-33.
47. Buffart TE, Carvalho B, Mons T, et al. DNA copy number profiles of gastric cancer precursor lesions. BMC Genom. 2007;8:345.

48. Fennerty MB. Gastric intestinal metaplasia on routine endoscopic biopsy. Gastroenterology. 2003;125:586-90.

49. Offerhaus GJ, Giardiello FM, Krush AJ, et al. The risk of upper gastrointestinal cancer in familial adenomatous polyposis. Gastroenterology. 1992;102:1980-2. 